\title{
Simple Correction of Alar Retraction by Conchal Cartilage Extension Grafts
}

\author{
Yong Jun Jang ${ }^{1}$, Sung Min Kim², Dae Hyun Lew ${ }^{1}$, Seung Yong Song ${ }^{1}$ \\ ${ }^{1}$ Department of Plastic and Reconstructive Surgery, Yonsei University College of Medicine, Seoul; ${ }^{2}$ Imi Plastic and Aesthetic Surgery Clinic, \\ Seoul, Korea
}

Background Alar retraction is a challenging condition in rhinoplasty marked by exaggerated nostril exposure and awkwardness. Although various methods for correcting alar retraction have been introduced, none is without drawbacks. Herein, we report a simple procedure that is both effective and safe for correcting alar retraction using only conchal cartilage grafting.

Methods Between August 2007 and August 2009, 18 patients underwent conchal cartilage extension grafting to correct alar retraction. Conchal cartilage extension grafts were fixed to the caudal margins of the lateral crura and covered with vestibular skin advancement flaps. Preoperative and postoperative photographs were reviewed and analyzed. Patient satisfaction was surveyed and categorized into 4 groups (very satisfied, satisfied, moderate, or unsatisfied). Results According to the survey, 8 patients were very satisfied, 9 were satisfied, and 1 considered the outcome moderate, resulting in satisfaction for most patients. The average distance from the alar rim to the long axis of the nostril was reduced by $1.4 \mathrm{~mm}$ (3.6 to 2.2 $\mathrm{mm}$ ). There were no complications, except in 2 cases with palpable cartilage step-off that resolved without any aesthetic problems.

Conclusions Conchal cartilage alar extension graft is a simple, effective method of correcting alar retraction that can be combined with aesthetic rhinoplasty conveniently, utilizing conchal cartilage, which is the most similar cartilage to alar cartilage, and requiring a lesser volume of cartilage harvest compared to previously devised methods. However, the current procedure lacks efficacy for severe alar retraction and a longer follow-up period may be required to substantiate the enduring efficacy of the current procedure.

Keywords Ear cartilage / Nose / Rhinoplasty / Transplants
Correspondence: Seung Yong Song Department of Plastic and Reconstructive Surgery, Yonsei University College of Medicine, 50-1 Yonsei-ro, Seodaemun-gu, Seoul 03722 , Korea

Tel: +82-2-2228-2210

Fax: +82-2-393-6947

E-mail: iceberg554@naver.com

Received: 9 May 2016 • Revised: 7 Sep $2016 \bullet$ Accepted: 20 Sep 2016

pISSN: 2234-6163 • elSSN: 2234-6171 • https://doi.org/10.5999/aps.2016.43.6.564• Arch Plast Surg 2016;43:564-569

No potential conflict of interest relevant to this article was reported.

\section{INTRODUCTION}

According to the Gunter classification of alar-columellar discrepancies, alar retraction is diagnosed when the distance between the alar rim and the long axis of the nostril is greater than $2 \mathrm{~mm}$ [1]. In this condition, the alar rim is retracted cephalad, exposing the nostril and creating an unnatural shape. An associated collapse of external nasal valves may have functional ramifications as well. Alar retraction in Caucasians is usually due to aggressive cephalic trimming of lower lateral cartilage during rhinoplasty. On the other hand, in East Asians, congenital hypoplasia of the lower lateral cartilage and alar skin, and cephalic 
malpositioning of the lower lateral cartilage are the primary causes of alar retraction $[2,3]$. Hence, it is ideal to bolster both cartilage and skin in East Asian patients with alar retraction.

At present, various procedures have been devised to correct alar retraction through composite grafts, grafting of cartilage, and soft tissue advancement [2,4-11]. In patients with mild-tomoderate alar retraction, alar contour grafts are effective. In moderate-to-severe states, alar spreader grafts, lateral crural strut grafts, and alar extension grafts are useful. Composite grafting is indicated for severe alar retraction $[5,12]$.

Unfortunately, such procedures are complex and unpredictable or have limited utility. Alar spreader grafts may produce a bulbous nasal tip; lateral crural strut grafting is not only complicated but also involves the possibilities of severe postoperative edema and worsening of alar flare; and alar contour grafts are restricted to mild alar retraction only. With composite grafts, estimating graft resorption is problematic, making it difficult to correct symmetrically. Donor site morbidity and hypertrophic scars are also probable.

In this study, we propose a simple procedure that is both effective and safe to correct alar retraction using only conchal cartilage graft.

\section{METHODS}

\section{Patients}

A retrospective review was conducted on 18 patients who underwent alar retraction correction with conchal cartilage extension graft between August 2007 and August 2009. The mean follow-up period was 11 months (range, 2-24 months).

\section{Preoperative patient characteristics}

Most alar retraction patients presented with alar notching and nostril exposure. The distance between the alar rim and the long axis of the nostril, measured in lateral photo views, ranged from 2.7 to $6.1 \mathrm{~mm}$, with a mean value of $3.6 \mathrm{~mm}$. Patients generally presented a flat nasal dorsum, limited nasal tip prominence, and reduced nasolabial angle $\left(<85^{\circ}\right)$. Plunging nasal tips (i.e., a long nose with severe caudal rotation) were evident in some. Alar retraction correction, nasal tip plasty, and augmentation rhinoplasty were concomitantly performed through an open approach.

\section{Surgical techniques}

Fig. 1 demonstrates the procedure of the operation. For an open approach, a marginal incision is made along the caudal border of the lower lateral cartilage. To ensure an adequate amount of vestibular skin flap to cover the extension graft without tension,

\section{Fig. 1. Diagram of conchal cartilage extension graft}

After creation of the vestibular skin advancement flap, the semilunar shape extension graft is fixed edge-to-edge with the caudal margin of the lower lateral cartilage.

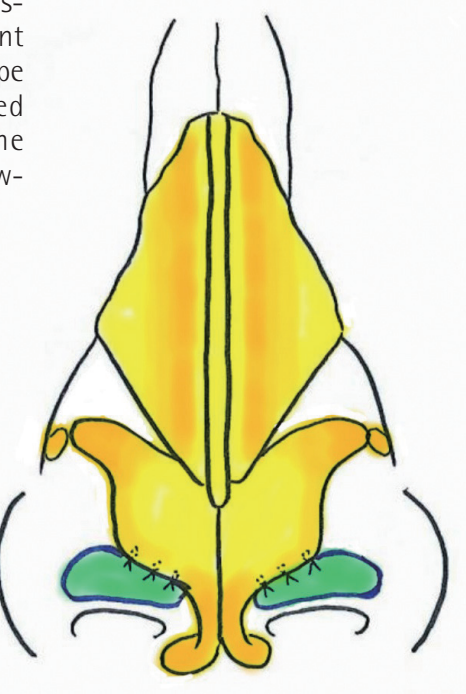

the marginal incision is tilted slightly cephalad.

A vestibular skin advancement flap is fabricated by dissecting the vestibular skin flap caudally from the marginal incision with Metzenbaum scissors (Fig. 2A). Before dissection, the alar vestibular skin is firm and tightly adhered, allowing no space for the extension graft. Sufficient dissection creates a stretchable vestibular advancement flap and enough space for the extension graft that enables effective and stable correction (Fig. 2B).

Semilunar shape cartilage is prepared by utilizing the $13 \times$ 6-mm-sized conchal cartilage harvested from the lateral concha.

The conchal cartilage extension graft is placed on the caudal margin of the lateral crus (Fig. 2C) and sutured 3 points edgeto-edge with 6-0 nylon (Fig. 2D).

For an additional stabilization of the extension graft, it is secured to the vestibular mucosa cephalic to the marginal incision with 1-point pull-out suture. Then, 5-0 Vicryl is used with the knot facing the side of the vestibular mucosa.

\section{Assessment}

Anteroposterior, semilateral, and lateral photos were taken before and after surgery for comparison. Through a patient survey, patient satisfaction was evaluated and was categorized into four groups: very satisfied, satisfied, moderate, and unsatisfied.

\section{RESULTS}

Our clinical series of patients $(\mathrm{n}=18)$ comprised 17 females and 1 male, whose mean age was 33 years old (range, 24-53 years), all of whom were East Asian. Alar retraction correction was combined with augmentation rhinoplasty, nasal tip plasty, 


\section{Fig. 2. Flap elevation and cartilage fixation}

(A) Alar vestibular skin is dissected caudally from the marginal incision. (B) Sufficient dissection creates a stretchable vestibular skin advancement flap that enables effective and stable correction. (C) Conchal cartilage is placed on the caudal margin of the lateral crus. (D) Conchal cartilage is fixed with a 3-point suture. The black arrow indicates the points of fixation.
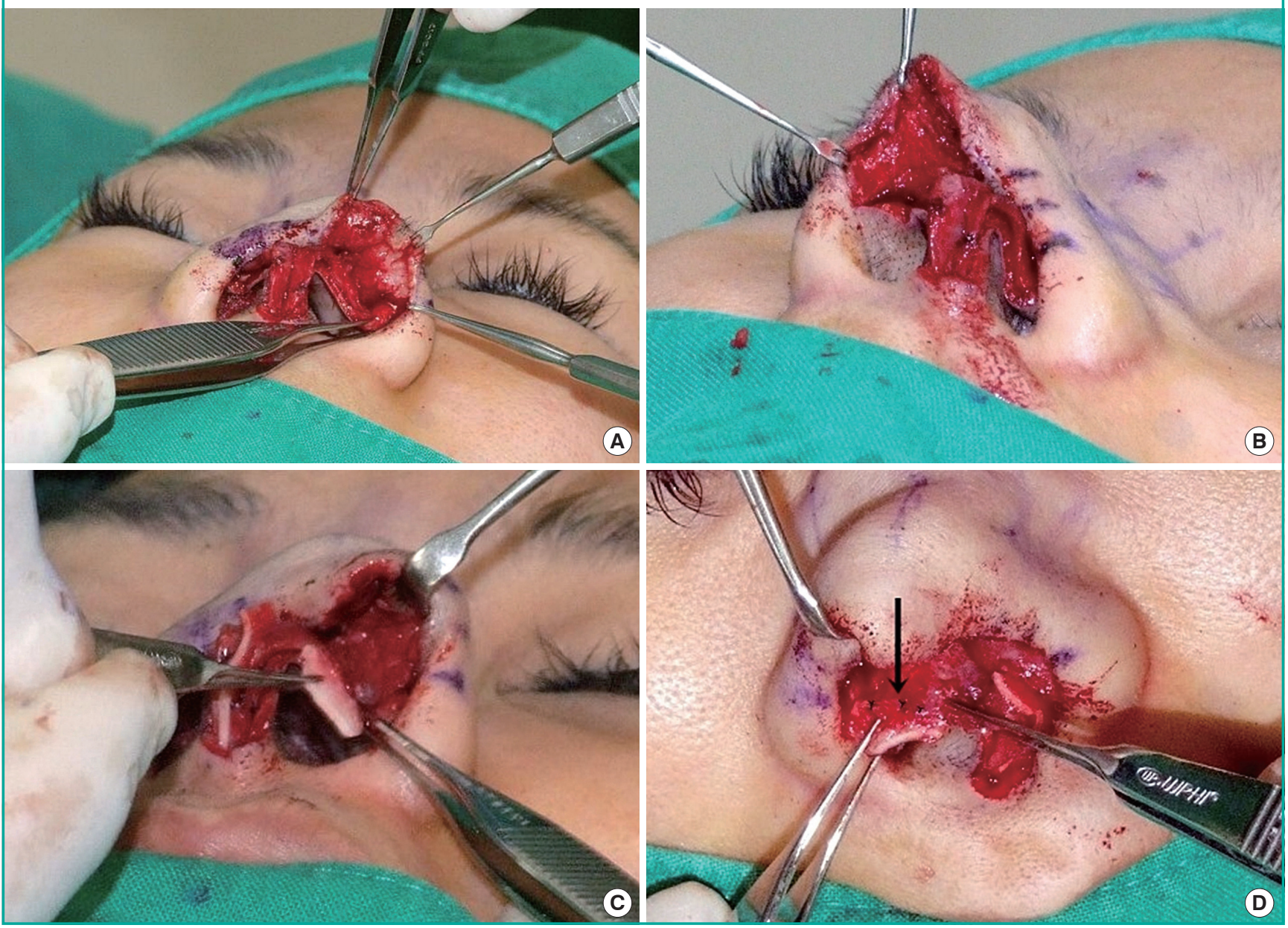

or secondary rhinoplasty in every patient.

According to patient survey responses, $44 \%$ of patients (8/18) were very satisfied, $50 \%(9 / 18)$ were satisfied, and $1 \%(1 / 18)$ had a moderate response, demonstrating satisfactory subjective results in patients. Patients were especially pleased with overall improvements in nasal shape, since correction of alar retraction and aesthetic rhinoplasty were combined. Through correction, alar notching improved to a smoother dome shape, and nostril exposure was reduced in every patient (Figs. 3, 4).

The average distance from the alar rim to the long axis of the nostril was reduced by $1.4 \mathrm{~mm}$ (range, 3.6 to $2.2 \mathrm{~mm}$ ) indicating an objective efficacy of the procedure.

Two patients experienced palpable cartilaginous step-offs that spontaneously resolved without any aesthetic problems. Alar retraction recurred in 1 patient who replied with a moderate result in the survey. No other specific side effects or complications were observed.

\section{DISCUSSION}

Because the alar rim plays a crucial role in the balance of the nasal base, deformities of the alar rim cause nostril exposure and undesirable nasal shape. Thus, for an aesthetically pleasing nose, an ideal alar-columellar relationship must be sought and obtained. Sheen and Sheen [4] have described an alar-columellar relationship as 2 to $3 \mathrm{~mm}$ of columellar show on lateral view. However, certain deformities such as hanging ala and retracted columella cannot be distinguished by this approach. Gunter et al. [1] described a more specific approach, classifying the alarcolumellar relationship into 6 types. According to this approach, the ideal distance from the long axis of the nostril to either alar rim or columella is within $1-2 \mathrm{~mm}$. In contrast, alar retraction is characterized by a distance from the long axis of the nostril to the alar rim greater than $2 \mathrm{~mm}$ and a distance from the long axis of the nostril to the columella within 1-2 mm [1]. Alar retrac- 


\section{Fig. 3. Patient illustration (severe alar retraction)}

A patient with severe alar retraction with distance from alar rim to long axis of the nostril originally $5.8 \mathrm{~mm}$ underwent correction with conchal cartilage extension graft. $(A, C, E)$ Preoperative views. ( $B, D$,

F) Postoperative views at 12 months.
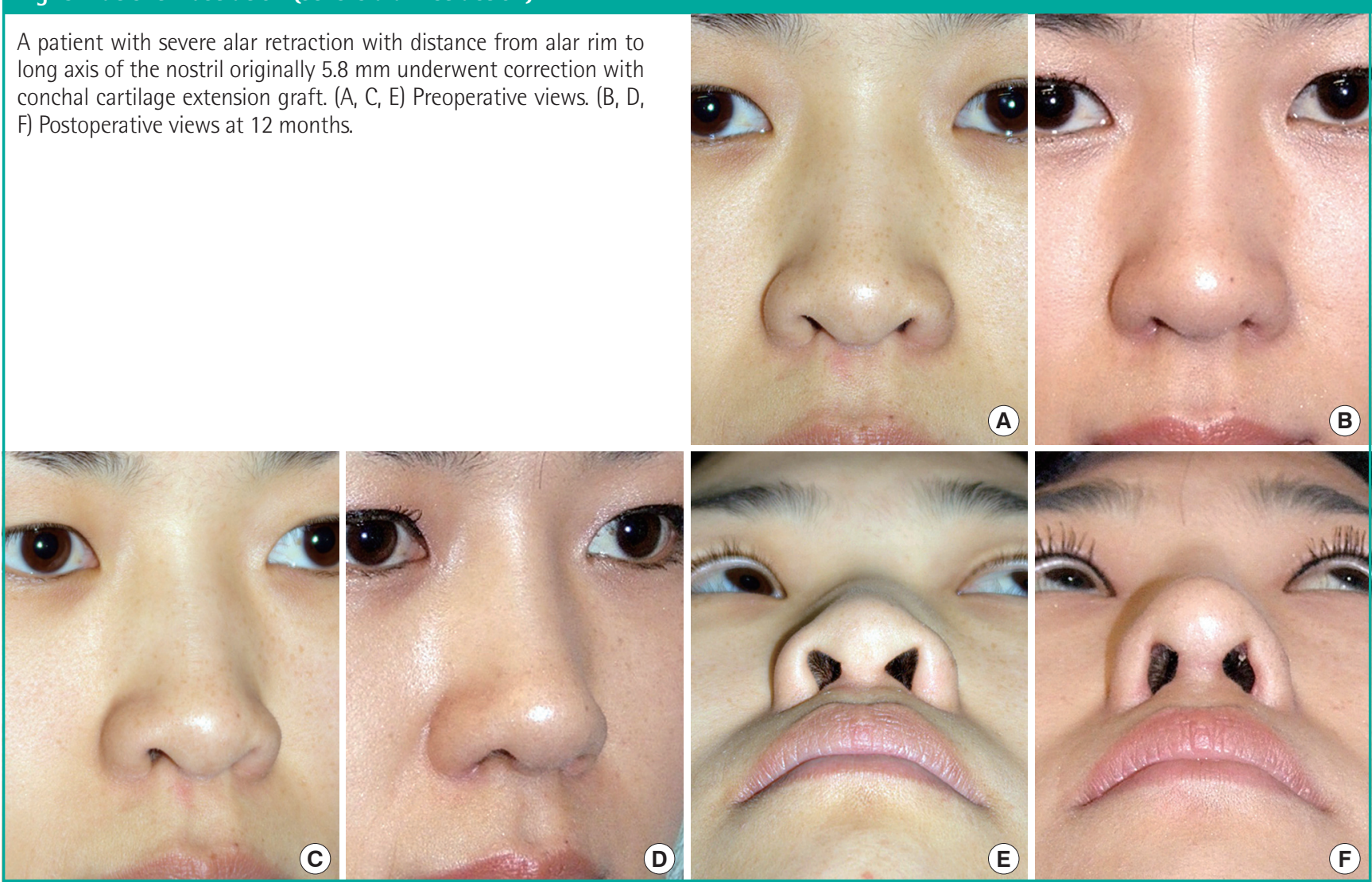

Fig. 4. Patient illustration (severe alar retraction)

A patient with severe alar retraction with distance from alar rim to long axis of the nostril originally $6.5 \mathrm{~mm}$ underwent correction with conchal cartilage extension graft. $(A, C, E)$ Preoperative views. $(B, D$, F) Postoperative views at 12 months.
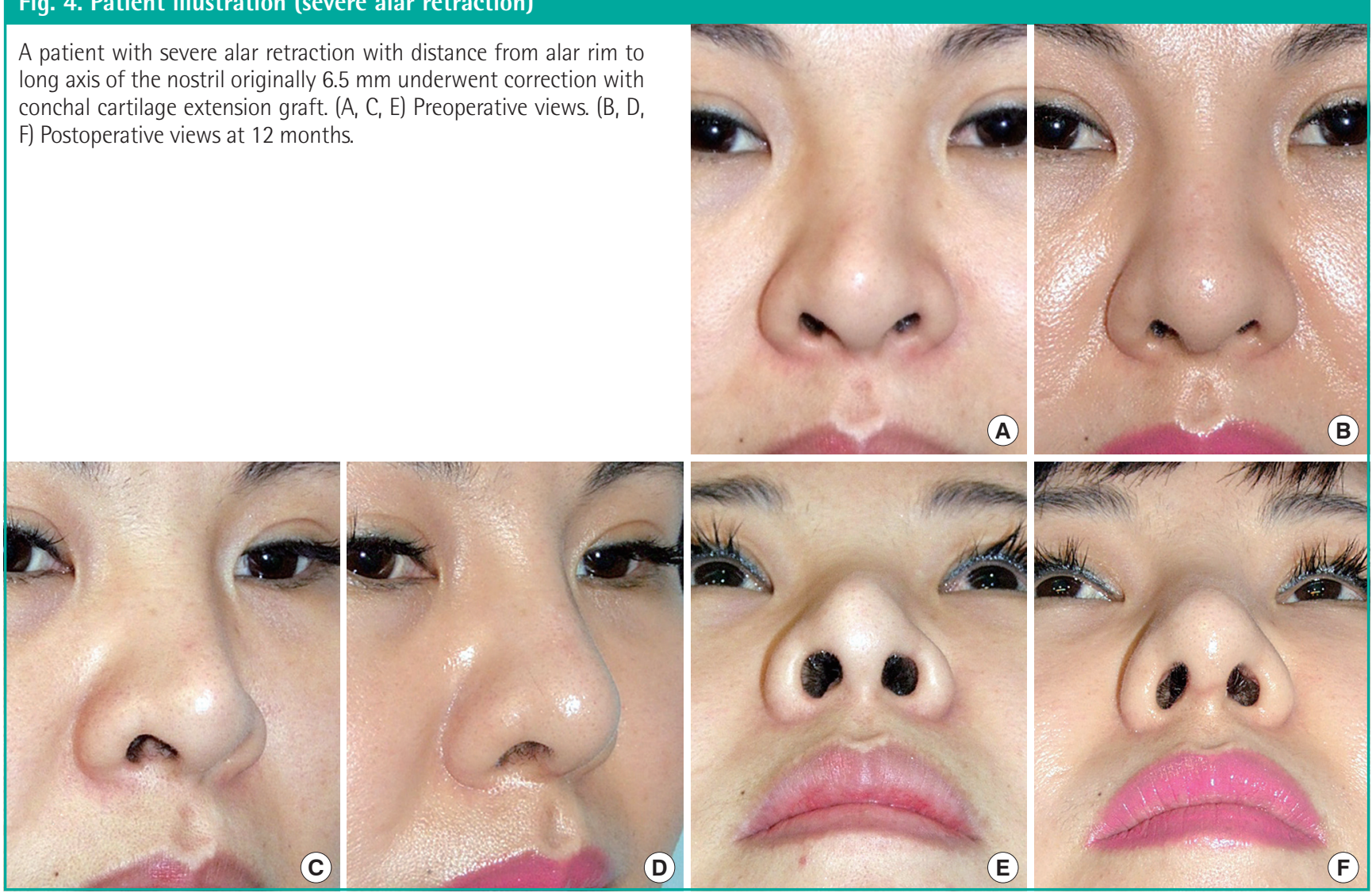
tion is typically diagnosed and corrected accordingly.

Unlike Caucasians, alar retraction in East Asian patients is mostly caused by congenital hypoplasia of the lower lateral cartilage and alar skin, and cephalic malpositioning of the lower lateral cartilage $[2,3]$. Our technique successfully addresses the congenital deficits of alar cartilage and skin encountered in East Asian patients by using conchal cartilage extension graft and a vestibular advancement flap together. Also, by tailoring the size of grafted cartilage, varying degrees of alar retraction are correctable, especially in mild-to-moderate states.

Using alar extension grafts to correct alar retraction was first described by Kim and Roh [6], originally harvesting septal cartilage for this purpose. Unlike nonanatomical alar contour grafts, alar extension grafts extend the alar rim anatomically. The medial edge of the triangular septal cartilage was fixed to the lateral crus, the lateral edge was tucked into a vestibular skin pocket, and a soft-shield graft was applied to prevent step-off at the alar margin.

Compared to the previous technique, conchal cartilage is used preferentially in our series, given that the intrinsic curvature of the conchal cartilage resembles that of the lateral crus of the lower lateral cartilage. Also, by suturing the extension graft edgeto-edge to the lower lateral cartilage, this method requires a lesser amount of cartilage and prevents bulging of the vestibular mucosa due to overlapping of the cartilage. Furthermore, the approach to vestibular skin differs. The previous method creates a vestibular skin pocket to fix the lateral end of the extension graft, which lacks alar skin reinforcement. In contrast, the current technique dissects the tightly adhered vestibular skin to form a vestibular advancement flap, which is flexible enough to cover the extension graft and lower the retracted alar rim. Since 3-point edge-to-edge fixation of the extension graft and lateral cartilage can be unstable, the extension graft and vestibular mucosa cephalic to the marginal incision are fixed with a 1-point pull-out suture for additional stability. When vestibular skin is dissected properly and the extension graft is secured robustly, it can adequately encounter the retraction force during wound healing, leading to lower recurrence of alar retraction. In addition, complications such as nostril asymmetry, visible step-off, and temporary paresthesia, all seen in the previous study, were not encountered in our cohort.

The distance between the long axis of the nostril and alar rim was reduced from 3.6 to $2.2 \mathrm{~mm}$ through our modified procedure, underscoring its efficacy. Nostril notching and exposure likewise were mitigated in every patient treated, with a $94 \%$ satisfaction rate claimed by patients. Aside from 2 cases of palpable cartilage step-off (both resolving spontaneously) and 1 case of recurrence, our patients were free of complications, such as ke- loid/hypertrophic scar formation, alar rim collapse, or airway obstruction.

The limitations of this study include its retrospective design, with a potential for selection bias. In addition, the postoperative follow-up period was relatively short. Postsurgical monitoring for $>1$ year is advisable to check for relapses of alar retraction. Finally, our patient population was small relative to those of the studies by Gunter and Friedman [10], Constantian [2], and Rohrich et al. [5]. Thus a larger prospective study with prolonged follow-up is warranted.

In conclusion, alar retraction correction with conchal cartilage alar extension graft is a simple, effective method of correcting alar retraction that can be combined with aesthetic rhinoplasty conveniently. Also, by employing conchal cartilage that is compatible to alar cartilage and vestibular advancement flap, it reinforces both alar skin and cartilage in alar retraction patients. In addition, edge-to-edge fixation of the extension graft and the lower lateral cartilage allows a lesser amount of cartilage required compared to previous methods.

\section{REFERENCES}

1. Gunter JP, Rohrich RJ, Friedman RM. Classification and correction of alar-columellar discrepancies in rhinoplasty. Plast Reconstr Surg 1996;97:643-8.

2. Constantian MB. Indications and use of composite grafts in 100 consecutive secondary and tertiary rhinoplasty patients: introduction of the axial orientation. Plast Reconstr Surg 2002;110:1116-33.

3. Kim JH, Park SW, Oh WS, et al. New classification for correction of alar retraction using the alar spreader graft. Aesthetic Plast Surg 2012;36:832-41.

4. Sheen JH, Sheen AP. Aesthetic rhinoplasty. 2nd ed. St. Louis: Quality Meical Publishing; 1998.

5. Rohrich RJ, Raniere J Jr, Ha RY. The alar contour graft: correction and prevention of alar rim deformities in rhinoplasty. Plast Reconstr Surg 2002;109:2495-505.

6. Kim HS, Roh SG. The alar extension graft for retracted ala. J Korean Soc Plast Reconstr Surg 2009;36:66-74.

7. Ellenbogen R. Alar rim lowering. Plast Reconstr Surg 1987; 79:50-7.

8. Constantian MB. The incompetent external nasal valve: pathophysiology and treatment in primary and secondary rhinoplasty. Plast Reconstr Surg 1994;93:919-31.

9. Gruber RP, Kryger G, Chang D. The intercartilaginous graft for actual and potential alar retraction. Plast Reconstr Surg 2008;121:288e-296e.

10. Gunter JP, Friedman RM. Lateral crural strut graft: tech- 
nique and clinical applications in rhinoplasty. Plast Reconstr Surg 1997;99:943-52.

11. Hirohi T, Yoshimura K. Surgical correction of retracted nostril rim with auricular composite grafts and anchoring sus- pention. Aesthetic Plast Surg 2003;27:418-22.

12. Alexander AJ, Shah AR, Constantinides MS. Alar retraction: etiology, treatment, and prevention. JAMA Facial Plast Surg 2013;15:268-74. 